\title{
Reflets
}

Revue d'intervention sociale et communautaire

\section{Réflexions du Regroupement pour la valorisation de la paternité sur le rapport du conseil de la famille et de l'enfance portant sur l'engagement des pères}

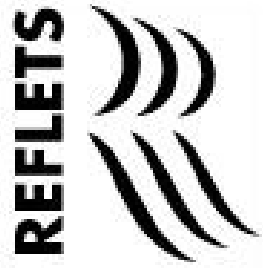

\section{Raymond Villeneuve}

Volume 15, numéro 1, 2009

Paternités : enjeux et perspectives (Première partie)

URI : https://id.erudit.org/iderudit/029595ar

DOI : https://doi.org/10.7202/029595ar

Aller au sommaire du numéro

Éditeur(s)

Reflets : Revue d'intervention sociale et communautaire

ISSN

1203-4576 (imprimé)

1712-8498 (numérique)

Découvrir la revue

Citer ce compte rendu

Villeneuve, R. (2009). Compte rendu de [Réflexions du Regroupement pour la valorisation de la paternité sur le rapport du conseil de la famille et de l'enfance portant sur l'engagement des pères]. Reflets, 15(1), 216-224.

https://doi.org/10.7202/029595ar 


\section{Réflexions du Regroupement pour la valorisation de la paternité sur le rapport du conseil de la famille et de l'enfance portant sur l'engagement des pères}

\section{Par Raymond Villeneuve}

directeur du RVP

Le 17 février dernier, dans le cadre de LATROISIÈME SU-PÈRE CONFÉRENCE, le Regroupement pour la valorisation de la paternité (RVP) rendait publiques ses réflexions sur le Rapport annuel 2007-2008 du Conseil de la famille et de l'enfance portant sur l'engagement des pères.

Considérant ce rapport comme un événement important dans l'histoire des politiques sociales en matière familiale au Québec, le RVP a cru essentiel de se livrer à une réflexion approfondie quant au contenu de ce document afin d'exprimer publiquement son adhésion à certains éléments du rapport, de remettre en question d'autres parties du document et finalement, de soumettre respectueusement aux différents niveaux de gouvernement des propositions afin que la contribution des pères soit mieux reconnue et que les pères en difficulté reçoivent un meilleur soutien. 


\section{Comité de réflexion}

Le comité de réflexion mis en place par le RVP était constitué des personnes suivantes: Dominique Arama (consultante pour le Département de la santé publique des Terres-Cries-de-laBaie-James, programme Strong and healthy children), Stéphane de Busscher, (intervenant à L'Hirondelle), Gilles Forget (chercheur, Direction de la santé publique Montréal-Centre), Yvon Lemay (intervenant à la Maison Oxygène), Manuel Prats (intervenant au CSSS Lucille-Teasdale), Gilles Rondeau (chercheur) et Raymond Villeneuve (directeur du RVP).

\section{Adhésion à certains éléments du rapport}

\section{Des statistiques éclairantes}

Le RVP est en accord avec le portrait sociétal présenté par le rapport. À ce titre, il nous semble intéressant de mettre en lumière certaines statistiques particulièrement révélatrices.

Par exemple, en 2006, 69 \% des hommes qui avaient droit au tout nouveau congé de paternité en ont profité. Pour le Conseil de la famille et de l'enfance, cela dénote un désir d'engagement des nouveaux pères. Par contre, entre 1998 et 2005, le temps alloué à la famille par les pères a diminué de plus de 5 heures par semaine en raison de l'augmentation du temps de travail et de déplacement. Cette situation crée une pression importante sur les papas.

Il est important de noter que la configuration des familles québécoises se modifie. Ainsi, en 2006, 14 \% des familles québécoises étaient recomposées et il y avait 68025 pères seuls, dont 39230 pères seuls avec au moins un enfant mineur; ce qui représentait près de $20 \%$ de l'ensemble des familles monoparentales. 
Il est intéressant de constater aussi que les gardes partagées sont en forte hausse au Québec puisqu'en 1995, elles représentaient seulement $11 \%$ des types de garde d'enfant privilégiés en cas de séparation ou de divorce, alors que cette proportion s'élevait à $29 \%$ en 2003.

Quant aux pensions alimentaires, il faut souligner que, dans environ $95 \%$ des cas, les pères sont les débiteurs à l'égard du versement de la pension et, qu'en mars 2006, la pension alimentaire avait été versée à temps et en entier dans $79 \%$ des cas.

Finalement, il nous semble très révélateur de mettre en lumière que, selon le rapport, 14,6 \% des pères en famille biparentale et $25 \%$ des pères en famille recomposée ressentent une détresse psychologique élevée. Malheureusement, aucune statistique n'a été présentée pour les pères seuls qui, vraisemblablement, subissent une détresse encore plus importante.

De nombreuses autres statistiques extraites du rapport pourraient être présentées ici, mais malheureusement, l'espace nous manque. Nous vous suggérons donc de consulter le document intégral disponible en ligne sur le site Internet du Conseil (www. cfe.gouv.qc.ca).

\section{Des constats importants}

Selon le comité de réflexion, la dimension la plus importante du rapport réside cependant dans les constats réalisés par le Conseil de la famille et de l'enfance. En effet, de nombreuses observations se révèlent porteuses de changements sociaux et il nous apparaît essentiel d'en reconnaitre la valeur.

\section{Situation des pères}

Le Conseil observe que les pères québécois s'impliquent davantage qu'avant auprès de leurs enfants et que cet engagement leur procure des bénéfices incontestables. Le Conseil constate aussi que les pères inadéquats sont une minorité et que les pères séparés ou divorcés sont plus impliqués qu'on le croit. Ces observations nous réjouissent puisqu'elles reflètent avec justesse et nuance la 
progression manifeste de l'engagement des pères québécois auprès de leurs enfants.

\section{Les difficultés des pères}

Le rapport souligne aussi que les difficultés des pères sont souvent minimisées lors des séparations et des divorces ou en situation de vulnérabilité. Ces assertions sont de la plus haute importance puisqu'elles rejoignent la perception du RVP voulant que la société québécoise, bien que souhaitant généralement un plus grand engagement des pères, tarde à reconnaître que ceux-ci peuvent subir aussi des difficultés, et ce, tout particulièrement lors des périodes de transition familiale. Le rapport souligne par ailleurs que l'accroissement du temps de travail crée un stress accru sur les pères et que les employeurs sont lents à s'adapter au nouveau rôle parental des hommes.

\section{Actions gouvernementales}

Quant aux actions gouvernementales, le rapport met en lumière un important manque de statistiques sur les pères, un manque de connaissance des actions du milieu communautaire pour ces derniers et un manque de financement général des actions dudit milieu. De plus, le Conseil de la famille et de l'enfance constate malheureusement que peu d'actions gouvernementales sont destinées spécifiquement aux pères. Finalement, le Conseil énonce que l'État devra se mettre à l'écoute des pères pour qu'ils deviennent véritablement des parents à part entière.

Pour illustrer cette situation, notons par exemple que le mot "pères" n'apparaît aucunement dans le Programme national de santé publique 2008-2012 alors que le mot "mères " y figure à de nombreuses reprises. On y traite donc de la santé, du bien-être et des conditions socioéconomiques des mères, mais pas de celles des pères. 


\section{Questionnements}

\section{Enjeux insuffisamment traités}

Le comité de réflexion constate que certains enjeux sont insuffisamment traités dans le rapport du Conseil de la famille et de l'enfance. Ainsi, il est très peu fait état des besoins des pères dans ce document qui arbore pourtant comme sous-titre : Rapport 2007-2008 sur la situation et les besoins des familles et des enfants.

Le comité tient à souligner aussi que plusieurs sujets sont peu approfondis dans le document. Voici les plus importants : la trajectoire des pères séparés ou divorcés et l'impact du processus judiciaire sur leur engagement; la trajectoire des pères immigrants; la situation spécifique des jeunes pères; les pères vivant en contexte d'exclusion; les pères autochtones; et aussi la réponse des services institutionnels et communautaires aux besoins des pères.

De même, le comité a noté l'absence d'une analyse des services sociaux et des difficultés vécues par les pères concernant ces services.

\section{Réserves}

Le comité a émis des réserves sur certains aspects du rapport du Conseil de la famille et de l'enfance. Le document énonce : «En somme, le genre ne détermine pas le rôle qu'on exerce auprès de l'enfant. " Tous les membres du comité ont tenu à signifier que, pour eux, il existait une spécificité quant à l'apport parental d'un père comparativement à celui d'une mère. De nombreuses études supportent d'ailleurs cette prise de position.

Le comité tient à souligner aussi qu'il est préoccupé par le fait qu'au Québec en 2009, un enfant peut naitre sans aucune filiation paternelle, et cela, dans plusieurs cas de figure. Cet état de fait n'est pas anodin selon nous, et l'État devrait se questionner quant à l'impact de ces dispositions sur les enfants des prochaines générations. 
Finalement, le comité tient à exprimer qu'il partage les questionnements de Germain Dulac à l'égard des études de temps. En effet, il nous semble évident que cet outil possède des limites importantes dont il faut tenir compte lors qu'on l'utilise : certaines tâches ne sont pas comptabilisées, la personne qui remplit le questionnaire a une perception subjective de son utilisation du temps, etc.

\section{Propositions aux gouvernements}

Le comité de réflexion a articulé des propositions qui seront adressées aux différents paliers de gouvernement afin que la contribution des pères soit mieux reconnue et que les pères en difficulté bénéficient d'un meilleur soutien. Le comité appuie aussi plusieurs propositions formulées par le rapport du Conseil de la famille et de l'enfance. Ces propositions se déploient autour de huit grands axes : recherche, formation, initiatives communautaires, offre de services du secteur de la santé et des services sociaux, politique sociale, conciliation travail-famille, réussite scolaire des jeunes garçons et campagne provinciale de promotion.

Voici le texte intégral de ces propositions :

\section{Recherche}

- Réaliser les efforts nécessaires afin de réduire l'écart existant en matière de données statistiques entre les pères, les mères et les enfants; (Le RVP appuie la proposition précédente du Conseil de la famille et de l'enfance.)

- Favoriser le développement de la recherche afin de mieux connaitre la situation des pères et les interventions qu'ils suscitent, renforcent et maintiennent leur engagement paternel.

\section{Formation}

- Favoriser l'intégration des réalités des pères dans les programmes de formation collégiale et universitaire des intervenants, et ce, 
tout particulièrement pour les programmes de travail social, de psycho-éducation, de médecine, de psychologue, de sciences infirmières, etc.;

- Favoriser la formation continue des intervenants des différents réseaux de services pour qu'ils puissent être en mesure de mieux rejoindre les pères et mieux répondre à leurs besoins;

- Favoriser la formation et l'intégration d'intervenants masculins dans les métiers et professions qui accompagnent les pères, les mères et les enfants.

\section{Initiatives communautaires}

- Réaliser un portrait des mesures en place s'adressant aux pères;

- Reconnaître l'importance du soutien aux pères dans l'offre de services à la famille;

- Financer adéquatement les initiatives de soutien aux pères.

\section{Offre de services du secteur de la santé et des services sociaux}

- Offrir aux pères les ressources présentes dans les milieux de services et de vie et recourir aux approches et aux manières de faire qui correspondent à leurs caractéristiques et à leurs attentes;

(Le RVP appuie la proposition précédente du Conseil de la famille et de l'enfance.)

- Mettre en place un programme national d'action auprès des pères, tout particulièrement pour ceux qui vivent en contexte d'exclusion.

\section{Politiques sociales}

- Réviser les lois et les mesures sociales pour qu'elles favorisent l'engagement des pères, que ce soit autour de la filiation, de la rupture, des contextes d'exclusion, des mesures d'aide sociale et des services sociaux; 
- Dans les pratiques des services de santé entourant la naissance, mieux prendre en considération les préoccupations des pères et mieux accompagner ces derniers au moment d'une transition des plus importantes pour eux et leur famille.

(Le RVP appuie la proposition précédente du Conseil de la famille et de l'enfance.)

\section{Conciliation travail-famille}

- Agir davantage, vigoureusement même, en faveur d'une meilleure conciliation des responsabilités familiales et professionnelles.

(Le RVP appuie la proposition précédente du Conseil de la famille et de l'enfance.)

\section{Réussite scolaire des jeunes garçons}

- Par conséquent, pour que les futurs pères soient capables de jouer envers leurs enfants le rôle qu'on attend d'eux, la réussite et la persévérance scolaires des garçons sont des objectifs à garder bien en vue.

(Le RVP appuie la proposition précédente du Conseil de la famille et de l'enfance.)

\section{Réaliser une campagne provinciale de promotion}

- Favoriser l'engagement des pères à l'aide d'une campagne sociétale portant sur leur implication et sur l'apport positif de cet engagement pour les hommes, femmes et enfants.

\section{Conclusion}

Le rapport annuel 2007-2008 du Conseil de la famille et de l'enfance portant sur l'engagement des pères constitue sans nul doute une étape importante dans l'avancement de la reconnaissance de ceux-ci comme des parents à part entière au Québec. 
Cependant, pour que la situation évolue véritablement, il faudra que des représentations soient réalisées auprès des différents paliers de gouvernement, que l'État se mette enfin à l'écoute des pères et qu'il se mobilise en leur faveur. Le RVP espère aussi que le Conseil de la famille et de l'enfance poursuivra son travail de manière à favoriser une meilleure intégration des réalités des pères dans l'offre de services à la famille.

Ces actions nous apparaissent essentielles parce que nous du RVP croyons profondément qu'une nouvelle vision de la famille doit émerger. Une vision où tous les membres de la cellule familiale sont également importants et reconnus dans leur spécificité. Nous sommes persuadés que c'est seulement lorsque cette nouvelle vision sera pleinement intégrée dans notre société que la véritable égalité entre les femmes et les hommes sera possible puisque c'est dans la famille que les hommes et les femmes de demain construisent aujourd'hui leur identité. 